\title{
A Generic Level 1 Simulator for Spaceborne GNSS-R missions and Application to GEROS-ISS Ocean Reflectometry
}

\author{
Hyuk Park, Senior Member, IEEE, Adriano Camps, Fellow, IEEE, Daniel Pascual, Student Member, IEEE, Yujin
} Kang, Raul Onrubia, Student Member, IEEE, Jorge Querol, Student Member, IEEE, and Alberto Alonso-Arroyo

\begin{abstract}
In the past decade Global Navigation Satellites System Reflectometry (GNSS-R) has emerged as a new technique for Earth remote sensing for various applications such as ocean altimetry and sea state monitoring. After the success of the GNSS-R demonstrator payloads aboard the UK-DMC or TDS1 satellites, at present there are several missions planned to carry GNSS Reflectometers. The GNSS rEflectometry, Radio Occultation and Scatterometry onboard International Space Station (GEROS-ISS) is an innovative ISS experiment exploiting GNSS-R technique to measure key parameters of ocean, land, and ice surfaces. For GEROS-ISS mission, the European Space Agency (ESA) supported the study of GNSS-R Assessment of Requirements and Consolidation of Retrieval Algorithms (GARCA). For this, it was required to accurately simulate the GEROS-ISS measurements including the whole range of parameters affecting the observation conditions and the instrument, which is called GEROS-SIM. To meet these requirements, the PAU/PARIS Endto-end Performance Simulator $\left(\mathbf{P}^{2} \mathbf{E P S}\right)$ previously developed by UPC BarcelonaTech was used as the baseline building blocks for the level 1 (L1) processor of GEROS-SIM. $P^{2}$ EPS is a flexible tool, and is capable of systematically simulating the GNSS-R observations for spaceborne GNSS-R missions. Thanks to the completeness and flexibility, the instrument-to-L1 data module of GEROS-SIM could be implemented by proper modification and update of $\mathrm{P}^{2}$ EPS. The developed GEROS-SIM was verified and validated in the GARCA study as comparing to the TDS-1 measurements. This paper presents the design, implementation, and results of the GEROS-SIM L1 module in a generic way to be applied to GNSS-R instruments.
\end{abstract}

Index Terms-GNSS reflectometry, altimetry, data simulation, simulator, GEROS-ISS

\section{INTRODUCTION}

$\mathbf{G}$ Lobal Navigation Satellite System Reflectometry (GNSS-R) is a relatively new passive remote sensing technique that measures GNSS opportunity signals reflected over the Earth's surface. It was proposed for mesoscale

Manuscript received ...

This work was supported by the Spanish Ministry of Economy and Competitiveness and FEDER EU under the project "AGORA: Tecnicas Avanzadas en Teledeteccion Aplicada Usando Senales GNSS y Otras Senales de Oportunidad"(MINECO/FEDER) ESP2015-70014-C2-1-R, and by the Agencia Estatal de Investigacin, Spain. Unidad de Excelencia Maria de Maeztu MDM2016-0600.

The authors are with the Remote Sensing Laboratory (RSLab), Department of Signal Theory and Communication (TSC), Universitat Politécnica de Catalunya BarcelonaTech (UPC), 08034 Barcelona, Spain, and also with the Institut d'Estudis Espacials de Catalunya (IEEC)/Universitat Politcnica de Catalunya (UPC), 08034 Barcelona, Spain (e-mail: park.hyuk@tsc.upc. edu; camps@tsc.upc.edu). altimetry [1], and recent studies on GNSS-R have yielded promising results on various remote sensing applications such as altimetry [2]-[4], sea state [5]-[14], ice [15]-[17], and soil moisture [18], [19]. Following the UK Disaster Monitoring (UK-DMC) [20], the TechDemoSat-1 (TDS-1) satellite lauched in 2014 [20]. The TDS-1 has acquired a large amount of spaceborne GNSS-R measurements. Recently Cyclone Global Navigation Satellite System (CYGNSS) launched , and it is expected to provide GNSS-R measurements. Currently, a spaceborne demonstrator mission has been proposed for altimetry applications [21], and others have been proposed for sea state monitoring [22]-[24]. Also there are studies for modeling and simulation for GNSS-R observables [25], [26].

The PAssive Reflectometry and Interferometry System (PARIS) altimeter was a GNSS-R instrument that combines bistatic radar and interferometry [27]. PARIS consisted of an uplooking and a down-looking antenna, to receive the direct and reflected signals from GNSS satellites. By cross-correlating them, all GNSS signals could be used including the restricted access GNSS ones. Since the full power spectrum of the GNSS signal can be exploited, the height precision of the instrument is maximized.

The PARIS concept has been used in the GNSS rEflectometry, Radio Occultation and Scatterometry onboard the International Space Station (GEROS-ISS). GEROS is an innovative experiment primarily focused on the exploitation of the reflected signals from the GNSS satellites at L-band to measure key parameters of ocean surfaces which are relevant to climate monitoring. Secondary mission goals are global atmosphere and ionosphere observations using the GNSS radio occultation techniques and the monitoring of land surface parameters using reflected GNSS signals.

In the framework of the development of these missions, it was necessary to simulate a generic GNSS-R spaceborne instrument. In other words, an end-to-end performance simulator had to be developed including the whole range of observation conditions, and instrument and platform parameters of these bistatic scattering measurements in order to better understand, analyze, and quantify the performance of this GNSS-R space mission. To meet the requirements, the Remote Sensing Laboratory at the Universitat Politècnica de Catalunya (UPC) had developed a specific software tool called the PAU/PARIS End-to-End Performance Simulator ( $\left.\mathrm{P}^{2} \mathrm{EPS}\right)$ [28], built on the experience of the SMOS End-to-end Performance Simulator (SEPS) for ESA [29]. 
The simulator has the following capabilities:

1) GNSS transmitting and receiving satellites orbit simulation,

2) Location of the specular reflection point and glistening zone (area where scattered power is collected),

3) Instrument modeling including instrument imperfections (antenna arrays, receivers, and correlator),

4) Sea surface scattering coefficient generation based on a global database of geophysical parameters,

5) Observables simulation: full Delay Doppler Maps (DDM) and waveforms (cut of DDM at a fixed Doppler shift) including observation geometry, obtained based on novel algorithm described in [30] highly optimized for computation time,

6) Graphical input/output user interface (GUI).

With these capabilities, a complete generic GNSS-R space mission can be accurately simulated in a efficient and convenient manner. Therefore, it can be used in a number of applications including spaceborne mission analysis and planning, instrument analysis and design, and geophysical parameter retrieval from GNSS-R observables, etc. The functional modules of $\mathrm{P}^{2}$ EPS had been reused to develop the GEROS Simulator (GEROS-SIM), especially for the module generating L1 data.

This paper presents the design and results of the current version of this simulator. First, the basic DDM modeling is discussed. As compared to conventional models, our DDM modeling presents several improvements in terms of accuracy and efficiency. Second, the simulation procedure is shown, and the implementation of each functional module is described one by one. The third part of this paper illustrates its performance using simulation and validation results. Finally, the application cases of the simulator are described with conclusions. This paper describes the general framework and modules of spaceborne GNSS-R instrument, which can be flexibly tailored according to the other application. The case of GEROS-ISS instrument is focused in this paper.

\section{BASIC MODELING AND SIMULATION PROCESS}

\section{A. DDM and Cross-Correlation Waveform (XCW) Modeling}

The most complete measurement of a GNSS-R instrument is the delay Doppler map, and its modeling is the core of the simulator. Conventionally, the DDM model is derived from bistatic radar observations, and it is expressed as [5], [31]:

$\left\langle\left|Y\left(\tau, f_{d}\right)\right|^{2}\right\rangle=\frac{P_{T} \lambda^{2}}{(4 \pi)^{3}} T_{i}^{2} \int_{A_{s}} \frac{G_{T}(\vec{\rho}) G_{R}(\vec{\rho})\left|\chi\left(\Delta \tau, \Delta f_{d}\right)\right|^{2}}{R_{R}^{2}(\vec{\rho}) R_{T}^{2}(\vec{\rho})} \frac{\pi|\Re|^{2} \text { in the spatial } q^{(x, y)}}{q_{z}^{4}}$ basic domain. For the convolution approach, the

where $P_{T}$ is the transmitted power, $G_{T}$ is the transmitter antenna gain, $G_{R}$ is the receiver antenna gain, $R_{T}$ and $R_{R}$ are the distances between the scattering point and the transmitter and the receiver, respectively; $T_{i}$ denotes the coherent integration time, and $\lambda$ is the electromagnetic wavelength of the signal; $\left|\chi\left(\Delta \tau, \Delta f_{d}\right)\right|^{2}$ stands for the square of the Woodward Ambiguity Function (WAF), which is usually split as the product of two functions, i.e., $\left|\chi\left(\tau, f_{d}\right)\right|^{2}=\Lambda^{2}(\tau)\left|S\left(f_{d}\right)\right|^{2}$. The delay offset and Doppler shift associated to the surface point $\vec{\rho}$ are represented by $\tau(\vec{\rho})$ and $f_{d}(\vec{\rho})$, respectively; $\Delta \tau=\tau-\tau(\vec{\rho})$, and $\Delta f_{d}=f_{d}-f_{d}(\vec{\rho}) ; \Re$ is the Fresnel reflection coefficient at a given polarization and incidence angle; $P\left(-\vec{q}_{\perp} / q_{z}\right)$ is the probability density function of sea surface slope; $\vec{q}$ is the scattering vector $\vec{q}=(2 \pi / \lambda)\left(\hat{n}_{i}-\hat{n}_{s}\right)$ where $\hat{n}_{i}$ and $\hat{n}_{s}$ are unit vectors in the incidence and scattering directions.

To improve the accuracy and time-efficiency, the following effects are considered for the $\mathrm{P}^{2}$ EPS observation model. First, the antenna array effect should be included. In a GEROS-ISS instrument, to enlarge the swath it is desirable to focus and track multiple glistening zones simultaneously, so the antenna array must be capable of beamforming and steering. The GEROS-ISS employs an hexagonal-type antenna array, and analog beamforming to generate four synthesized beams [27]. Therefore, $\mathrm{P}^{2}$ EPS should be able to calculate the synthesized beam pattern of an antenna array configured in terms of the number of elements, the antenna spacing, and the element's weight (windowing). Then, the receiving antenna gain $G_{R}(\vec{\rho})$ in (1) is replaced by the term of synthesized beam pattern $|A F(\vec{\rho})|^{2}$, which includes the elementary antenna pattern.

Second, the impact of receiver hardware must be taken into account. The GNSS-R instrument processes the reflected signals collected by the down-looking antenna through the receiver chain, and finally cross-correlates them with either replicas of the GNSS code (in the conventional GNSS-R) or with the direct signals collected by the up-looking antenna (in the interferometric GNSS-R as the PARIS IoD). Thus, the measured DDMs are affected by the frequency response of the receiver chains and the correlator performance. These receivers and correlator effects are somehow similar to the fringewashing function effects in a synthetic aperture radiometer, and can be equivalently represented as a decorrelation function [32]. The measured DDM can then be computed as the convolution of the ideal DDM with a fringe-washing function

Additionally, an efficient DDM calculation method must be devised for a spaceborne mission simulator. To obtain simulated DDMs using (1), the functions inside the integrand are calculated over a very large number of spatial points $\vec{\rho}$. Certainly, this approach becomes too much time and resource consuming for spaceborne mission simulations because the size of the observed surface (glistening zone) extends over hundreds of kilometers. In [30], an efficient computation was proposed, which uses two dimensional convolution in the delay-Doppler $\left(\tau, f_{d}\right)$ domain instead of the double integrals $\left\langle\left|Y\left(\tau, f_{d}\right)\right|^{2}\right\rangle=\left[\frac{P_{T} \lambda^{2} T_{i}^{2}}{(4 \pi)^{3} R_{R}^{2} R_{T}^{2}}\right]\left|A F\left(\tau, f_{d}\right)\right|^{2} \Sigma^{o}\left(\tau, f_{d}\right) * * \chi^{2}\left(\tau, f_{d}\right) * \mid \tilde{r}_{e q}($

where $\Sigma^{o}\left(\tau, f_{d}\right)$ denotes the product of scattering coefficient and its Jacobian determinant as described in [30]. Similarly, receiver effects are included in the fringe-washing function denoted by $\tilde{r}_{e q}(\tau)$ [32], and a noise term $N\left(\tau, f_{d}\right)$ is added.

The $\mathrm{P}^{2}$ EPS DDM model in (2) shows well the items to be included in the simulation, and the process itself. As compared 
to the conventional model in (1), the DDM model equation of $\mathrm{P}^{2} \mathrm{EPS}$ (2) allows to simulate additional terms for antenna array beamforming $A F\left(\tau, f_{d}\right)$, and receiver and correlator effects $\tilde{r}_{e q}(\tau)$. Furthermore, the DDM calculation is expressed as a convolution form, and consequently the DDM model of $\mathrm{P}^{2}$ EPS allows more efficient simulation.

The XCW $w(\tau)$ is a one-dimensional function resulting from the correlation of the direct and reflected signals, after constant Doppler frequency compensation, so that it passes usually through the peak of DDM. i.e.,

$$
w(\tau)=\left.\left\langle\left|Y\left(\tau, f_{d}\right)\right|^{2}\right\rangle\right|_{f_{d}=\text { const }} .
$$

Hereafter $w(\tau)$ is referred to as a sample waveform.

\section{B. Incoherent Averaging}

To reduce the noise, individual sample waveforms are incoherently averaged as

$$
w_{\mathrm{avg}}(\tau)=\frac{1}{N_{a}} \sum_{k=1}^{N_{a}} w_{k}\left(\tau-\Delta \tau_{k}\right),
$$

where $N_{a}$ denotes the number of sample waveforms incoherently averaged, and $\Delta \tau_{k}$ means the Delay Difference Change (DDC) for $k$ th sample waveform. The delay difference between the direct and reflected signals changes due to the satellite motions. To properly average the waveform samples, this DDC should be compensated in the tracking and retracking procedures [33], [34]. Hereafter $w_{\text {avg }}(\tau)$ is referred as an averaged waveform.

The propagation of GNSS signals is also affected by the ionosphere and troposphere, e.g., additional delay, attenuation, scintillation, Faraday rotation, etc. These ionospheric and tropospheric effects should be included in the waveform simulation, and then be removed (compensated) in the height estimation.

\section{Simulation Process and Structure Design}

Using (2), the simulation procedure is as follows:

1) Determination of the observation geometry: The states (position and velocity) of GNSS transmitting and receiving satellites at a given epoch are computed in the Earth reference frame, Earth-centered Earth-fixed (ECEF). Using the computed positions, the specular point is found.

2) Glistening zone definition: Among the specular reflection points, the ones with an incidence angle smaller than a predefined value (e.g. smaller than $35^{\circ}$ ) are selected, and the glistening zone is defined. The number of delay and Doppler shift bins around the specular point with a uniform resolution $\Delta \tau$ and $\Delta f_{d}$ are defined. From a $\left(\tau, f_{d}\right)$ grid, the surface point $\vec{\rho}\left(\tau_{n}, f_{d, m}\right)$ is obtained.

3) Calculation of Ionospheric and Tropospheric effects: Once the positions of the GNSS, receiver satellites, and the specular points are obtained, the ionospheric and tropospheric effects can be calculated based on models and the environmental auxiliary parameter inputs (e.g., total electron content, total ground pressure, etc.).

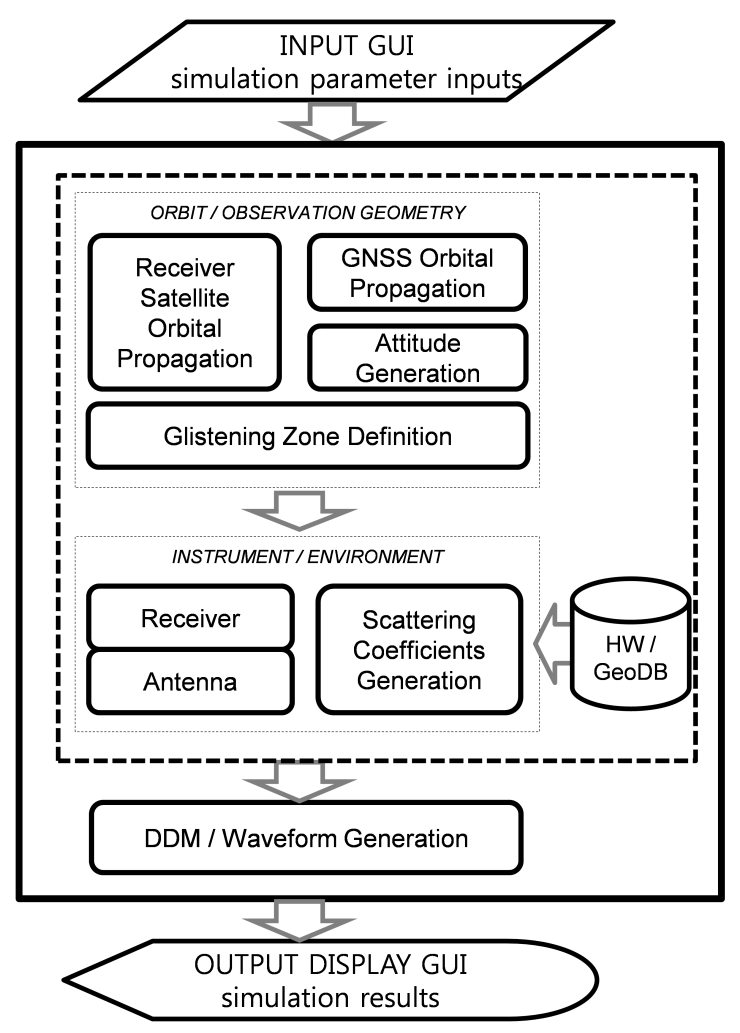

Fig. 1. Simulator structure diagram.

4) Computation of the antenna beam pattern (amplitude and phase): The beam pattern $A F\left(\tau, f_{d}\right)$ is computed considering the array configuration and the elementary antenna beam patterns.

5) Computation of the scattering coefficients: The scattering coefficients of the surface points are computed based on the geophysical database. Additionally, the values of the Jacobian determinants are multiplied by the scattering coefficients to produce $\Sigma^{o}\left(\tau, f_{d}\right)$ in (2).

6) Generation of the WAF and the fringe-washing function: The WAF is generated in the $\left(\tau, f_{d}\right)$ variables to perform the convolution. The fringe-washing function $\tilde{r}_{e q}(\tau)$ is also computed based on the receiver's frequency responses.

7) Calculation of a DDM by multiplication and convolution.

8) Inclusion of noise contribution (thermal and speckle parts).

9) Incoherent averaging.

For a systematic implementation of the simulator, the above processes are designed as separate functional modules. The structure is shown in Fig. 1. From the next section, the function and implementation of the modules are described in detail.

\section{Functional Module Implementation}

The modules of $\mathrm{P}^{2} \mathrm{EPS}$ have been implemented and integrated on Matlab ${ }^{\circledR}$ for the purpose of efficient implementation and maintenance of the simulator code. In this section, the implementation of different modules is described in detail. 


\section{A. Satellite Orbit and Field of View (FOV)}

This module estimates the states (position and velocity) of the GNSS transmitting and the receiving satellites. Once the simulation epoch is set, the states of transmitting GNSS satellites (Tx) are determined, for example, using orbital elements read from a Two-Line Elements set (TLE) (available from http://celestrak.com). In parallel, the state of the receiving satellite $(\mathrm{Rx})$ is obtained under the user-specified predefined orbit. With the positions of the GNSS and the receiver satellites, the specular point is computed in the ECEF system, and the corresponding incidence angle is also found. The attitude of the receiver satellite can also be defined. For the GNSS$\mathrm{R}$ observation, the attitude of the receiver satellite is usually set to local normal pointing (nadir looking) and the beam is electronically steered in the specular reflection direction. The RPY (roll, pitch, yaw) angles can also be used to set the receiver satellite attitude.

After finding the three points of the observation geometry (positions of the transmitter, the receiver, and the specular reflection point), the FOV around the specular point is defined as a regular grid in the $\left(\tau, f_{d}\right)$ domain, i.e., iso-range and iso-Doppler grid. Finding the scattered points $\vec{\rho}\left(\tau_{n}, f_{d, m}\right)$ is usually the computational bottleneck. In [30], an efficient numerical method to find these points is given, and this method has been revised and optimized for improved computational speed [28]. After finding the scattered points in the FOV, the corresponding Jacobians $\left|J\left(\tau_{n}, f_{d, m}\right)\right|$ are calculated. This observation geometry can be computed more efficiently by transforming of the reference frame from the ECEF to the SRF (Scattering Reference Frame) shown in Fig. 3 in [33]. The details of SRF definition is explained in [33].

The uncertainty in the knowledge of the observation geometry can be introduced in the Tx and Rx states, and the sea height. When the uncertainties of $\mathrm{Tx}$ and $\mathrm{Rx}$ positions and velocity are given, for example, as a form of error probability density function (PDF), the states of $\mathrm{Tx}$ and $\mathrm{Rx}$ can be produced with errors. Likewise, the error of sea height can also be introduced, and then the position of the specular point, the Doppler frequency of direct and reflected signals have uncertainties.

The data and processing flow of this module are illustrated in Figure 2.

\section{B. Scattering Coefficient}

This module generates the term $\Sigma^{o}\left(\tau, f_{d}\right)=$ $\sigma^{o}\left(\tau, f_{d}\right)\left|J\left(\tau, f_{d}\right)\right|$ in (2) over the FOV. First, the scattering coefficient $\sigma^{o}\left(\tau, f_{d}\right)$ is calculated using the model in [5] which is based on the KA-GO (Kirchhoff Approximation-Geometric Opctics):

$$
\sigma^{o}=\frac{\pi|\Re|^{2} q^{4}}{q_{z}^{4}} P\left(-\frac{\vec{q}_{\perp}}{q_{z}}\right) .
$$

The method is widely used for GNSS-R scattering model of sea surface because of its simplicity and good accuracy around the specular reflection direction. The conditions of the observation scene are applied through the sea surface slope PDF $P(\vec{s})$ where $\vec{s}=-\vec{q}_{\perp} / q_{z}$. In [35], the sea surface slope PDF is described using a Gram-Charlier distribution which is function of mean-square-slope (MSS) of up-wind $m s s_{u}$ and cross-wind $m s s_{c}$. The Gram-Chariler distribution introduces skewness and peakdness in the sea surface slope PDF by modification of tow dimensional Gaussian distribution [36]. For the GNSS signal, the MSS described in [35] is suitable for optical band, which are the overestimated value for microwave L band. The equivalent MSS for GNSS signal is reported by [37]:

$$
\begin{aligned}
& m s s_{u}(U 10)=0.45 \times\{0.000+0.00316 \cdot f(U 10)\}, \\
& m s s_{c}(U 10)=0.45 \times\{0.003+0.00192 \cdot f(U 10)\},
\end{aligned}
$$

where $f(U 10)$ is defined by

$$
f(U 10)= \begin{cases}=U 10, & 0.00<U 10 \leq 3.49 \\ =6 \ln (U 10)-4.0, & 3.49<U 10 \leq 46 \\ =0.411 \cdot U 10, & 46<U 10 .\end{cases}
$$

The sea surface wind speed is denoted by U10 (m/s).

The whole range of geophysical parameters affecting the scattering coefficient are used including sea surface temperature, salinity, and surface roughness expressed by the wind speed (zonal and meridional components).To increase the accuracy of the simulation, the same geophysical database constructed for SMOS End-to-end Performance Simulator (SEPS) [29] is used, which provides the global maps of various kinds of geophysical parameters including their monthly variability. The computed scattering coefficients are then multiplied by the corresponding Jacobians to obtain $\Sigma^{o}\left(\tau, f_{d}\right)$. The data flow and processing of the scattering coefficient generation module is illustrated in Figure 3.

\section{Ionospheric / Tropospheric Effects}

In order to precisely estimate the delay paths of the reflected (from down-looking antennas) and the direct (from up-looking antennas), the propagation path characteristics and their effects should be analyzed. They should also be modeled and included in the E2E performance simulation. The main impact in the propagation path is due to the troposphere and the ionosphere, and results in the additional delay. Especially for the spaceborne altimetry application of GEROS-ISS, the modeling of this effect is important. However, this ionospheric effects are negligible in some applications, e.g. scatterometry application. In this section, these two effects and their models are briefly described, and the way to include in the simulation is described.

1) Ionospheric effects on GNSS signal propagation: The ionosphere is a layer of electrons and electrically charged atoms and molecules that surrounds the Earth, stretching from a height of about $50 \mathrm{~km}$ to more than $1,000 \mathrm{~km} \mathrm{[38].} \mathrm{The}$ free electrons in the ionosphere affect the propagation of radio waves. At GNSS signal band (L-band), radio waves passing thought the ionosphere are affected in amplitude, phase, and speed. For the attenuation effect, it is negligible for GNSS-R performance simulation. For example, the maximum attenuation is $0.0082 \mathrm{~dB}$ for a vTEC (vertical total electron content) of 80 TECU (total electron content unit) at mid latitude. Another effect is scintillation in amplitude and phase. For E2E simulation, the scintillation also discarded because it 


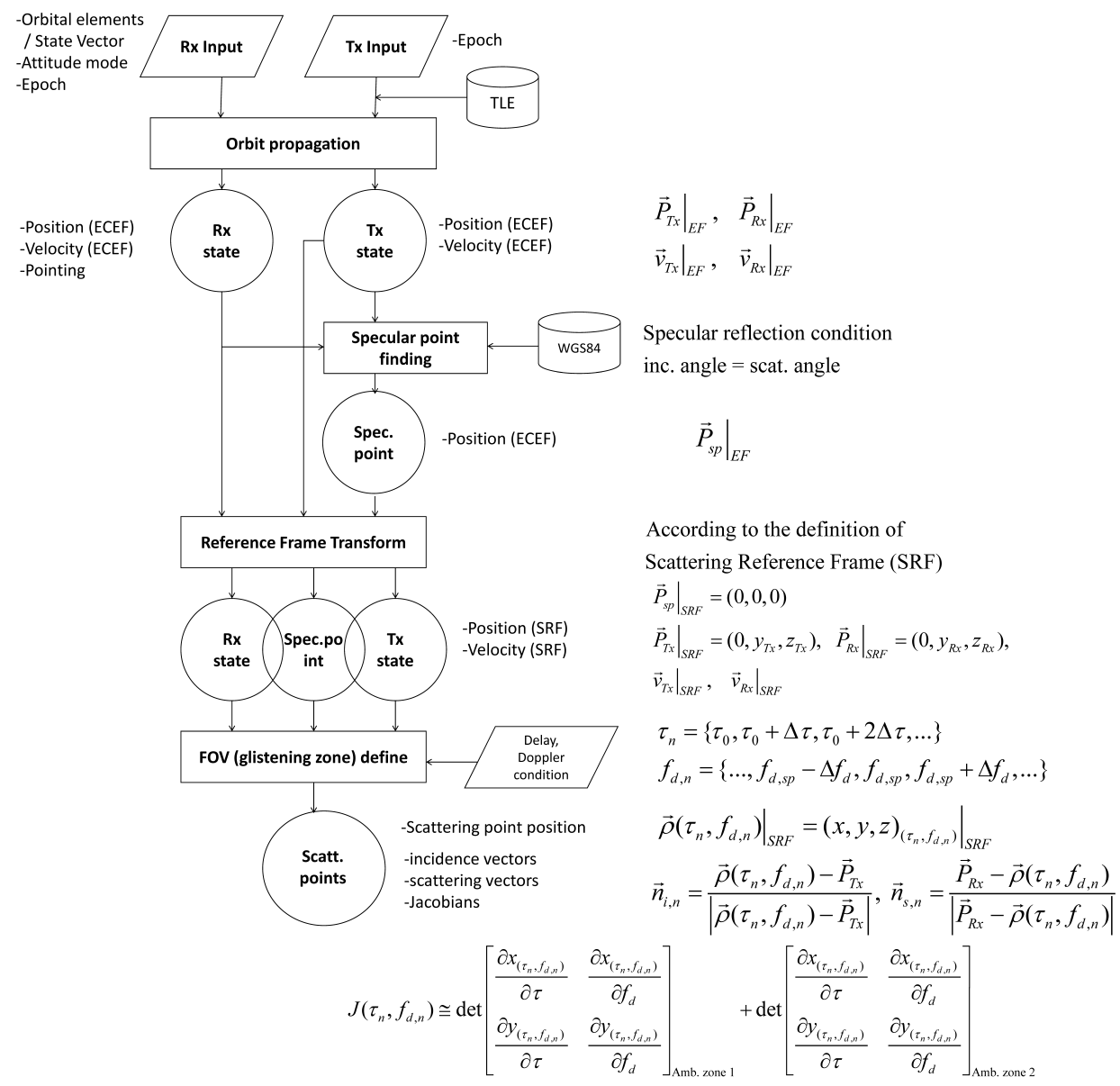

Fig. 2. Data and processing flow of the orbit and FOV module.

is very localized (temporarily and regionally), and expected to have a negligible impact on the total performance [27], [39]. Only exceptional case is when the reflection takes place over very calm water surface for which the coherent component of reflection is dominant [39].

The main effect of the ionosphere is the group delay affecting the PRN (Pseudo Random Noise) signal used to modulate the carrier. The ionospheric delay is dependent on the signal frequency and the amount of electron contents through propagation path, and it is usually given by

$$
\rho_{\text {ion }}=\frac{40.35}{f_{c}^{2}} \text { sTEC, }
$$

where $f_{c}^{2}$ is carrier freqeuncy $(\mathrm{Hz})$, ans $s T E C$ denotes the slant total electron content related to the vTEC as sTEC $=$ vTEC $/ \cos (\chi)$, and:

$$
\cos (\chi)=\sqrt{1-\left(\frac{R_{E} \cos (E)}{R_{E}+h_{p p}}\right)^{2}},
$$

In [40], the geometry for the ionospheric delay model is shown with the meaning of notations and variables in (9); the Earth's radius $R_{E}$, the height of ionosphere shell (defeind as pierce point in [40]) $h_{p p}$. For example, when the vTEC is $80 \mathrm{TECU}$, the elevation angle of the GNSS satellite (E) is $55^{\circ}$, the ionospheric delay of GPS L1 signal $(1575.42 \mathrm{MHz})$ is calculated to be $15.82 \mathrm{~m}$ (= $52.73 \mathrm{~ns})$ from GNSS satellite to the specular reflection point, and therefore, the reflected signal received by the down-looking antenna is delayed by 105.47 ns as compared to the case of propagation in the vacuum. For the direct signal received by the up-looking antenna, the ionospheric delay can also be calculated based on the sTEC from the transmitting satellite to the receiving one. In order to simulate the waveform including the ionospheric delay, the simple model (8) can be used to calculate the ionospheric delay for each band, and then add the ionospheric delay in each coherent waveform. For the vTEC model, the global ionospheric map can be used [41].

2) Tropospheric effects on GNSS signal propagation: As the ionosphere, the troposphere also affects the GNSS signal propagation by inducing the delay, attenuation, and scintillation. Among them, the attenuation (atmospheric and rain attenuation), and the scintillation can be neglected at L-band. The atmospheric attenuation is about $0.035 \mathrm{~dB}$ at zenith, and it is negligible [42]. Rain attenuation is also very small at Lband; even for an intense rainfall (e.g., $100 \mathrm{~mm} / \mathrm{hr}$ ), it is less than $0.01 \mathrm{~dB} / \mathrm{km}$. Scintillation can also be discarded for the same reason as in the ionospheric case. Therefore, only the tropospheric delay is considered in the simulation. The signal 


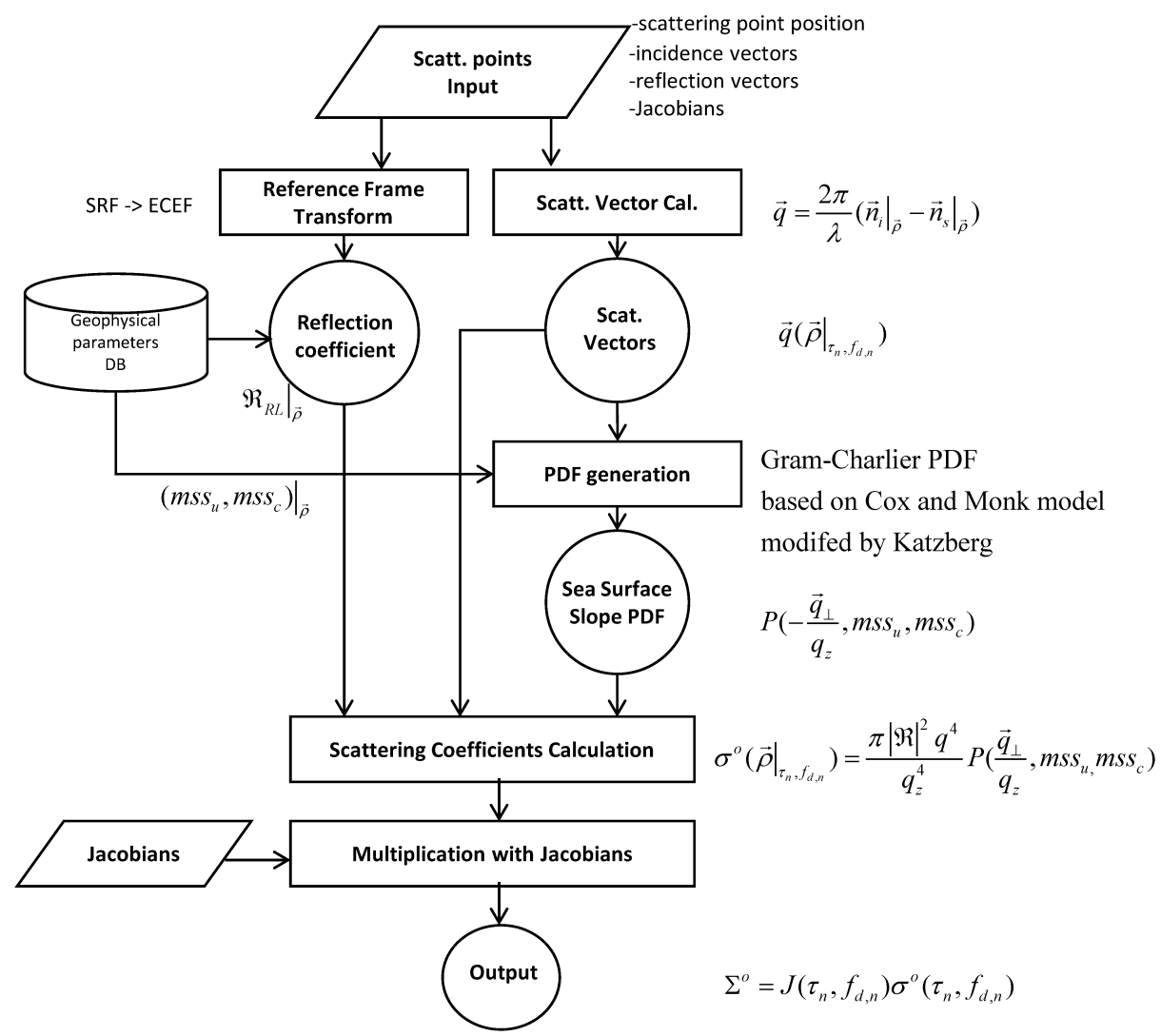

Fig. 3. Data and processing flow of the scattering coefficient generation module.

received from GNSS satellite is refracted by the atmosphere on or near the Earths surface. Atmospheric refraction causes an additional delay and depends on the actual path of the curved ray, and the refractive index of the gases along that path. There are several models of the tropospheric refraction, and the corresponding delay for GNSS signals [42]. For the direct problem, it is common to first consider the tropospheric delay for a zenith path, and apply the mapping function corresponding to the elevation angle. For a zenith path, it is convenient to consider the tropospheric delay separately by the hydrostatic and wet delays [43]:

$$
\Delta L=\Delta L_{h y}^{z} m f_{h y}(\gamma)+\Delta L_{w e t}^{z} m f_{\text {wet }}(\gamma)[\mathrm{m}],,
$$

where $\Delta L_{h y}^{z}$ is the hydrostatic zenith delay; $m f_{h y}(\gamma)$ is the hydrostatic zenith delay mapping function; $\Delta L_{\text {wet }}^{z}$ is the wet zenith delay; and $m f_{\text {wet }}(\gamma)$ is the wet zenith delay mapping function. For the mapping function, the simple model $m f_{h y}(\gamma)=1 / \sin (\gamma)$ can be used. The hydrostatic (dry) zenith delay is modeled as [42] :

$$
\begin{aligned}
\Delta L_{h y}^{z} & =\left(0.0022768 \pm 5 \cdot 10^{-7}\right) \frac{P_{o}}{g(\text { lat }, H)}[\mathrm{m}] \\
g(\text { lat }, H) & =1-0.00266 \cos (2 \text { lat })-2.8 \cdot 10^{-7} \times H
\end{aligned}
$$

where $P_{o}$ is the total ground pressure (hPa); lat is the latitude in degree; and $H$ is the height above ellipsoid in $\mathrm{m}$.
The dry component of the tropospheric delay is about $90 \%$ of the total delay, and it corresponds to approximately $2.3 \mathrm{~m}$ in the zenith direction and varies with the local temperature and atmospheric pressure [42]. The wet component is generally smaller, from a few $\mathrm{mm}$ in dry arctic areas or in deserts, to 40 $\mathrm{cm}$ in tropical regions [44]. Since the water vapor profile is difficult to obtain using only ground-based measurements of the temperature, pressure and humidity, the wet tropospheric delays remains usually unsatisfactory estimated by any a priori model, especially when high accuracy positioning and tropospheric parameters are targeted. Therefore, in simulations, the wet zenith delay is selected randomly in the range of 1 to 30 $\mathrm{cm}$, and kept constant in the simulation of coherent waveforms (individual waveform samples) for single averaged waveforms. Another approach is to use a more complex model for total tropospheric delay [42]:

$\Delta L=\frac{0.002277}{\sin \gamma}(2-g($ lat,$H))\left[P_{o}+\left(\frac{1255}{T_{o}}+0.05\right) e_{o}\right](\mathrm{m})$,

where $P_{h y}$ is the partial pressure of hydrostatic air (hPa); $e_{o}$ partial pressure of water vapor $(\mathrm{hPa}) ; T_{o}$ is the surface temperature $(\mathrm{K})$.

For example, the tropospheric delay is $2.94 \mathrm{~m}$ in the case that the elevation angle $\gamma=55^{\circ}$, latitude lat $=30^{\circ} \mathrm{H}=1000$ $\mathrm{m}, P_{h} y=1000 \mathrm{hPa}, e_{o}=13.25 \mathrm{hPa}$. Therefore, the reflected 
signal received by down-looking antenna is delayed by 5.87 $\mathrm{m}$ (19.58 ns) as compared to the case of propagation through vacuum. For the direct signal received by up-looking antenna, the tropospheric delay is negligible.

In order to compensate for the tropospheric delay, the double differencing method proposed in [27] can be used, i.e., difference of reflected-minus-direct delay observations at two different positions mesoscale processing, for short ground baselines, where the paths that cross through the troposphere can be considered homogeneous. Otherwise, on post-processing using correction models assimilated by ground meteorological observations to improve accuracy.

\section{Instrument Effects}

The simulation allows the definition of the array configuration and beamforming, which is represented as a beam pattern $A F\left(\tau, f_{d}\right)$ in (2). For example, the GEROS-ISS use a rectangular type with hexagonally positioned antenna array (see Fig. 8 in [21]) to generate multiple highly directive beams that can be steered to track the glistening zone [21].

The beamforming is achieved by phase compensation and summation, as in a conventional phased array. Therefore, the synthesized beam pattern $A F\left(\tau, f_{d}\right)$ can be expressed as

$A F\left(\vec{\rho}\left(\tau_{n}, f_{d, n}\right)-\vec{\rho}\left(\tau_{F O}, f_{d, F O}\right)\right)=\sum_{m=1}^{N_{a n t}} W_{m} W_{m}^{e} D_{m} \exp \{-j 2$

where $\vec{\rho}\left(\tau_{F O}, f_{d, F O}\right)$ is the surface point focused by array, $\tau_{F O}$ denotes the delay from the focusing point to the antenna, $W_{m}$ is the window function, $D_{m}$ is the $m$ th elementary antenna beam pattern, $N_{a n t}$ is the number of elementary antennas, and $f_{0}$ is the carrier center frequency. For the elementary antenna beam pattern $D_{m}$, the simulator uses data files corresponding to a simple L-band patch antenna. Once the elementary antenna patterns of actual instrument will be measured, they can be used for simulation. in actual instrument. Actual beamforming circuitry is not ideal, so it suffers from quantization errors in gain and phase shifter. In (13), $W_{m}^{e}$ represents the impact of random residual errors in beamforming: [45]

$$
W_{m}^{e}=\left(1+A_{m}^{e}\right) \exp \phi_{m}^{e},
$$

where $A_{m}^{e}$ and $\phi_{m}^{e}$ are the amplitude and the phase errors introduced by the $m$ th chain of beamformer. These errors cause the slight deformation of the shape of beam, usually resulting in increased sidelobe level as discussed in [45]. These errors with elementary antenna beam pattern also contribute to the beam pointing error. In the simulator, the standard deviations of the amplitude and phase errors are set to 0.25 $\mathrm{dB}$ and $2^{\circ}$, respectively, and these values can be modified at any time. The data flow and processing of the antenna module is illustrated in Fig. 4.

The analysis of the receiver frequency response and crosscorrelation effects on the GNSS-R observables has been described in [32], and it can be expressed as the one dimensional convolution of the DDM with the fringe-washing function for a constant Doppler shift. In other words, the impact of the receiver frequency response is a decorrelation or a widening of the DDM in the delay direction. The frequency response is computed either using a numerical computation as in [46], or using actual measured frequency responses. Through this, the characteristics and the receiver imperfection effects, for example the different bandwidth effects are included.

The data flow and processing of the receiver module is illustrated in Fig. 5.

\section{E. DDM / Waveform Computation}

The DDM computation module performs the multiplication and convolution including all the previously evaluated terms. First, the WAF is generated according to the observation mode. The WAF is usually split as the product of $\left|S\left(\Delta f_{d}\right)\right|=\left|\sin \left(\pi \Delta f_{d} T_{i}\right) / \pi \Delta f_{d} T_{i}\right|$ and the auto-correlation function (ACF) $\Lambda(\tau)$ of the code. In conventional GNSS-R, the reflected signal is correlated with a locally generated replica of the pseudo-random noise (PRN) code of the received signal. For the GPS C/A code, $\Lambda(\tau)$ is given by

$$
\Lambda(\tau) \simeq\left\{\begin{array}{lr}
1-|\tau| / \tau_{c} & |\tau| \leq \tau_{c}, \\
0 & \text { otherwise }
\end{array}\right.
$$

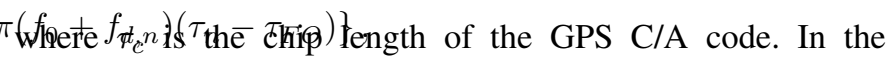
interferometric mode like in PARIS IoD, the reflected signal is correlated with the directly received signal from the uplooking antenna. In this case, $\Lambda(\tau)$ is more complicated than the conventional case because it uses the full bandwidth of all the signals present in that part of the spectrum [27]. For example, in the GPS L1 band, all codes (C/A and P, as well as $\mathrm{M}$ code if present) are cross-correlated, and then $\Lambda(\tau)$ is a composite form corresponding to the characteristic of each code:

$\Lambda_{L 1}(\tau)=\frac{P_{L 1 C A} \Lambda_{L 1 C A}(\tau)+P_{L 1 P(Y)} \Lambda_{L 1 P(Y)}(\tau)+P_{L 1 M} \Lambda_{L 1 M}(\tau)}{P_{L 1 C A}+P_{L 1 P(Y)}+P_{L 1 M}}$

where $P_{L 1 C o d e N a m e}$ and $\Lambda_{L 1 C o d e N a m e}$ denote the power, and ACF of the corresponding CodeName of GPS L1 band. Fig. 6 shows the shape of $\left|\Lambda_{L 1}(\tau)\right|^{2}$ in (15) and (16). Beside using 16 , the WAF is generated based on the signals spectrum and modulation type described in [48]. In the [48], the waveforms of various of GNSS signals are illustrated in detail, including GPS L5, Galileo E1, and E5.

Instead of the model WAF, a more practical version of the WAF of GNSS signals can be applied. For example, the small ripples outside the main lobe of WAF can be modeled, can be directly applied to the generation of DDM. Likewise, the WAFs of various GNSS signal, such as Galileo E1, E5 can be applied, when their cross-correlation shapes would be obtained analytically or experimentally. In the simulator, the WAF is generated either model or using real measured one in [47]. After generating the WAF, the noise-free DDM is computed by multiplication and convolution of the components previously computed. 


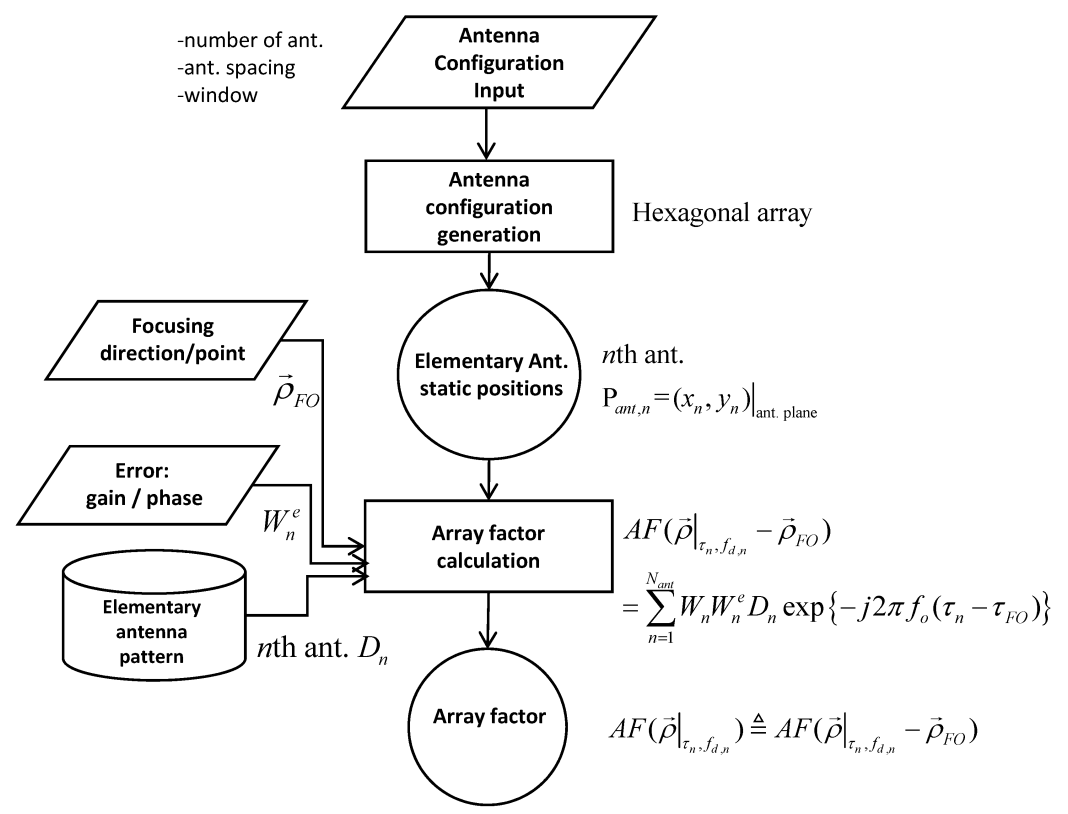

Fig. 4. Data and processing flow of the antenna module.

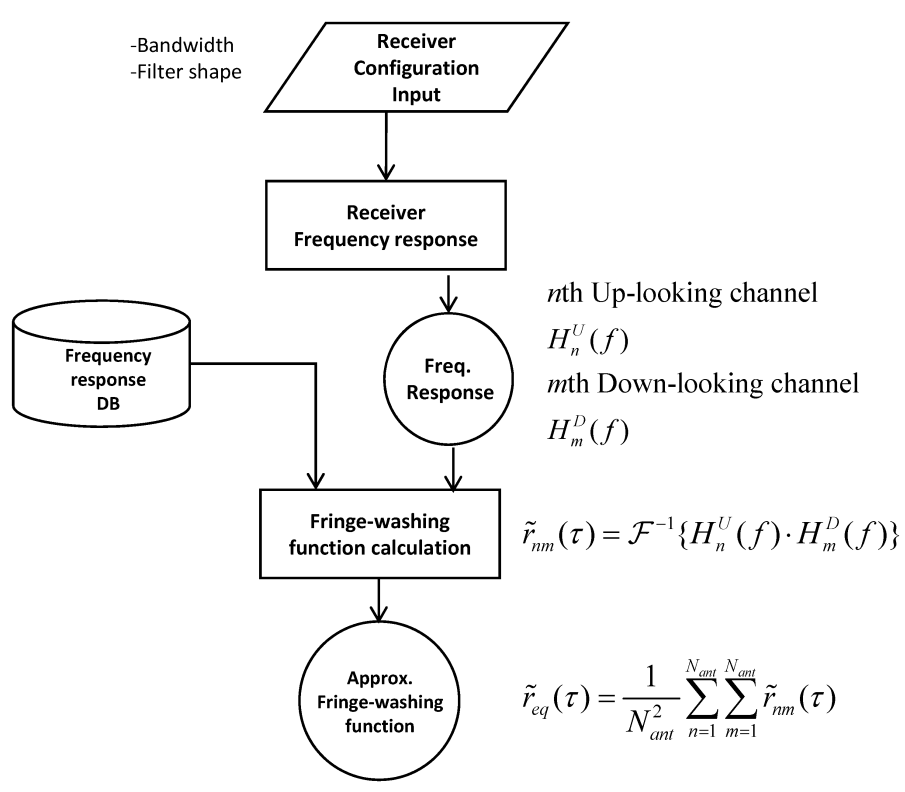

Fig. 5. Data and processing flow of the receiver module.

\section{F. Noise Effect}

In GNSS-R observable DDM / Waveform, two types of noise component can be considered: 1) thermal and 2) speckle noise. The noisy DDM is modeled as

$$
\begin{aligned}
Y_{n}\left(\tau, f_{d}\right) & =\sqrt{D D M_{C L}\left(\tau, f_{d}\right)}\left[\alpha+\frac{1}{\sqrt{2}}\left\{n_{i s}\left(\tau, f_{d}\right)+j n_{q s}(\tau,\right.\right. \\
2 A_{m}^{2} & =k\left(T_{a n t}+T_{o}(F-1)\right) B_{w}
\end{aligned}
$$

where $D D M_{C L}\left(\tau, f_{d}\right)$ is the clean DDM equivalent to(1); $n_{i s}$, $n_{q s}, n_{i t}$, and $n_{q t}$ is random variables with standard normal distribution; and $\alpha$ is the portion of coherent component relative to the incoherent one, in the received signal. For the case of GEROS-ISS ocean reflection, $\alpha$ is quite small, and in the GERSO-SIM simulation $\alpha$ is assumed to be 0 for ocean reflection. The thermal noise power is taken into account via $A_{m}$ where $k$ is Botzman's constant, $T_{a n t}$ is the antenna temperature, $T_{o}$ is the receiver temperature, $F$ is the noise figure, and $B_{w}$ is receiver bandwidth. The noise model (17) is based on the fact that speckle is 1) multiplicative noise, i.e., it is directly proportional to the signal's power (DDM/waveform value), and 2) signal and noise are statistically independent each other. As in synthetic aperture radar (SAR) imaging, speckle noise is caused by the coherent summation of the signals from elementary scatterers within the instrument resolution [49], [50]. Each of the diffuse scatterers contributes with a component to the echo signals in a sum known as a random walk in the complex plane. In fully developed speckle, this complex echo signal from diffuse scatterers alone has a zero mean two-dimensional Gaussian probability density function (PDF) in the complex plane. Therefore, the absolute value of this complex speckle noise has a Rayleigh distribution.

Another speckle property is its spatial correlation, i.e., the speckle appears as the same as the pixel size of the imaging system, which is found in SAR imaging [49], [50]. This spatial correlation is also found in the UK-DMC / TDS-1 measurements, and therefore it should be properly modeled. Usulally, it is modeled by convolution of system function (or In GNSS-R case, $\mathrm{WA}^{2} \mathrm{~F}^{5}$ ) with randomly generated noise. In the GERO-SIM, the noise model (17) implemented in an efficient way, shown in Fig. 8. 

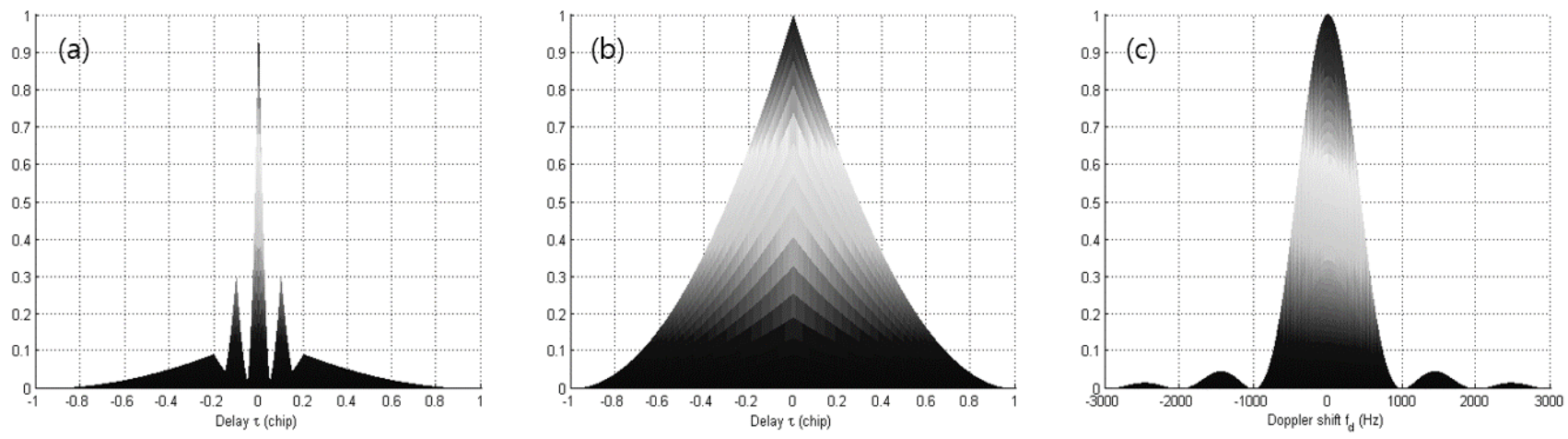

Fig. 6. Woodward ambiguity function used in P2EPS simulation: (a) $|\Lambda(\tau)|^{2}$ of PARIS interferometric mode using full L1 band of GPS signal, (b) $|\Lambda(\tau)|^{2}$ of conventional GNSS-R mode using C/A code in GPS L1 band, (c) $\left|S\left(\Delta f_{d}\right)\right|^{2}$ for 1 ms coherent integration time.

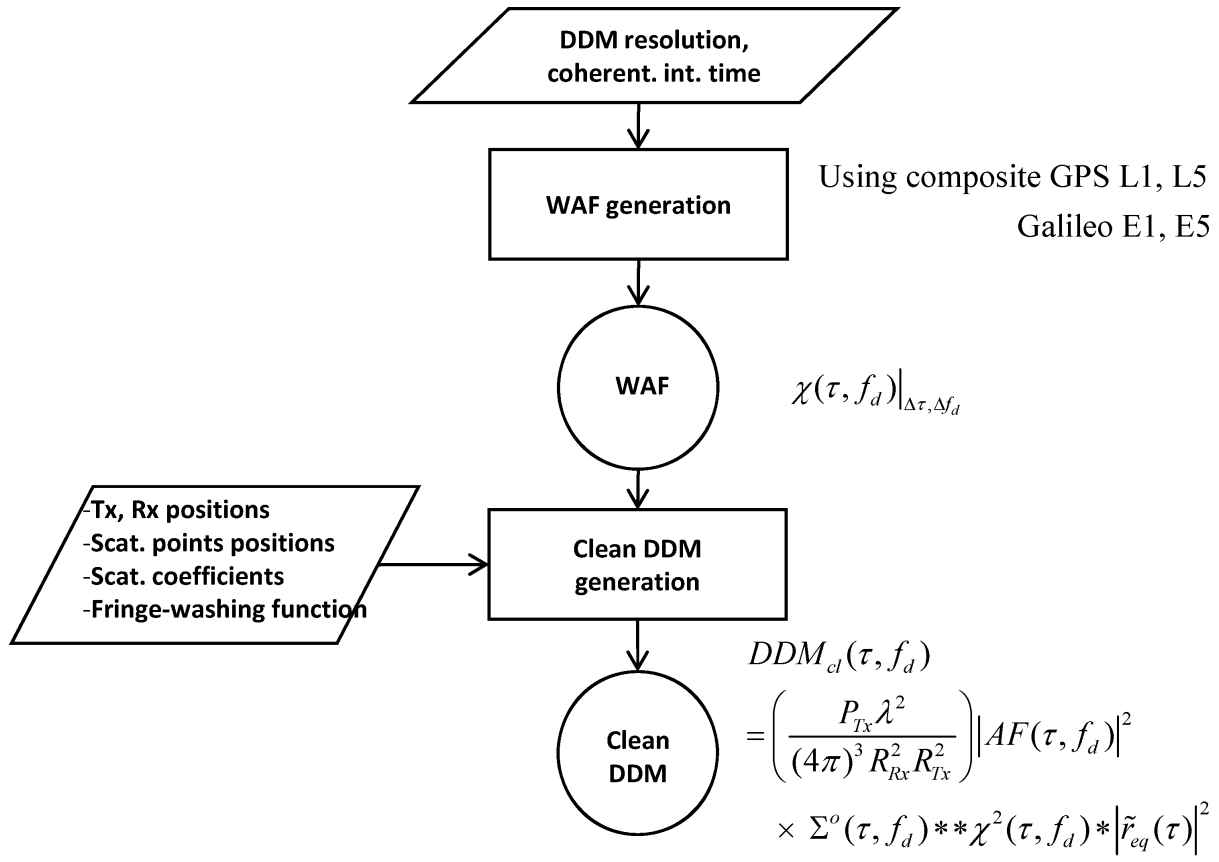

Fig. 7. Data and processing flow of the DDM generation module.

\section{G. Incoherent averaging}

Through the process described in above, the coherently integrated DDMs (waveforms) are obtained. For the incoherent averaging, the coherently integrated DDMs (waveforms) are successively generated corresponding to the observation geometry changes in the orbit propagation. All the time varying parameters and random process are applied to the coherent integrated waveform samples. If the number of waveform samples is given by $N_{\text {avg }}$ (or incoherent integration time is given by $T_{a v g}$ ), the simulation parameters are refreshed in each waveform samples, until obtaining the $N_{\text {avg }}$ samples.

After the simulation of the waveform samples, the incoherent integration (averaging the waveform samples) is conducted in order to reduce the noise and improve the waveform shape. The actual delay domain of the waveform samples is not the propagation time domain, but the index of the lag (or index of the tracking gate), which is usually set with the reference of the direct signal arrival time. Therefore, in order to properly put the significant part of waveform (e.g., leading edge, and peak), the waveform tracking is required. In other words, for the proper average of the waveform samples, $\Delta \tau_{k}$ in (4) is estimated for each waveform samples, and compensated (alignment of waveform samples) before averaging. Otherwise, the shape of waveforms are seriously distorted, and eventually the altimetric performance is degraded [33]. The GEROS simulation also should be employed the tracking procedure. According to the proposal of the open-loop tracking, the $\Delta \tau_{k}$ is pre-calculated and compensated for incoherent averaging. The more detail tracking considerations are discussed in [34]. 


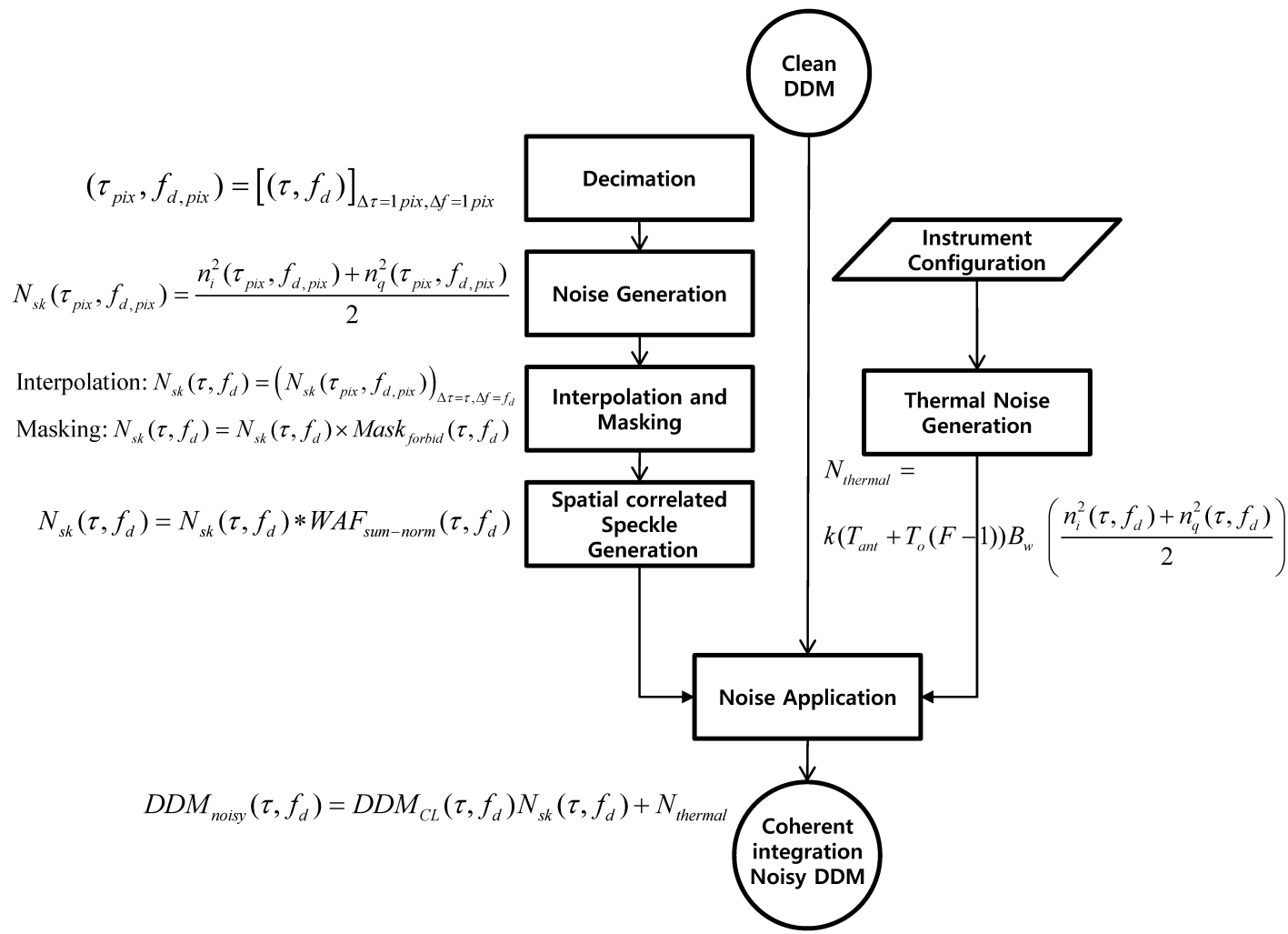

Fig. 8. Data and processing flow of the DDM generation module.

\section{Simulator Performance and Validation}

As in any simulator, validation tests have to be performed in various conditions and input parameters to show that the results obtained in known conditions are properly reproduced. Through the tests, accuracy and computation speed of the simulator has been evaluated as follows:

\section{A. Simulation Performance}

The accuracy has been evaluated by comparing the DDM values obtained by the simulator and by the conventional model in (1). The DDM modeling are mainly based on the method described in [30], which has shown equivalent results to the conventional method. Therefore, the accuracy of the simulator also corresponds to that result. The conventional model has been proved to have very similar results obtained with other algorithms, such as the facet approach [51] .

\section{B. Simulation verification and validation}

The GEROS-SIM L1 simulator has been constructed using the $\mathrm{P}^{2} \mathrm{EPS}$ as main building block. The $\mathrm{P}^{2} \mathrm{EPS}$ has been validated comparing to the spaceborne UK-DMC data and airborne data [55], [56]. Because the lack of real GEROS-ISS measurements, other available spaceborne GNSS$\mathrm{R}$ data (TDS1 data) are used for GEROS-SIM validation. For GARCA GEROS-SIM validation, the TDS-1 data used in [57] is also used. They are acquired over ocean between September 2014 and February 2015. For the collocated wind speed data, the ASCAT wind speed data withing $1 \mathrm{~h}$ temporal and 1 degree spatial location. From those data, total 57 set of DDMs has been selected where the wind speeds of data set are in the range of $1-18 \mathrm{~m} / \mathrm{s}$. For the verification and validation test, the antenna gain of TDS-1 was selected as higher than 10.1 dBic because the behavior of TDS-1 DDM is not stable. To verify the variations three main input parameters are selected: elevation angle, wind speed, antenna gain. And, to validate the algorithm, 57 cases of TDS-1 DDMs are collected with variation of those three parameters: antenna gain ranging (10.1 - $13.3 \mathrm{dBic})$, and elevation angle $\left(70.7^{\circ}-89.1^{\circ}\right)$. From these set, it is possible to test the dependency of simulated DDM on the parameters (wind, antenna gain and elevation angle).

The GEROS-SIM L1 data generation are set up and run in a configuration as similar as possible to the characteristics of the TechDemoSat-1 mission and GPS-R data, for example in terms of altitude $(825 \mathrm{~km})$ and DDM size and resolution $(0.25$ C/A chip in delay, $100 \mathrm{~Hz}$ in Doppler), receiver bandwidth, and antenna pattern available in [20]. All the detail information for validation test conditions are presented in [56].

Examples of the validation test results are shown in Fig. 9, and all the 57 cases are presented in [58]. The GEORSSIM L1 simulator generates the L1 data (DDM/ Waveforms, and aux/ancillary data), with the various input. Therefore, according to the variation of input parameters, the produced L1 data should vary (Verification) and the aspect of variation should be meet with the real data (Validation).

The results showed the good agreements between simulated 
TABLE I

DATA SPECIFICATIONS AND SIMULATION PARAMETERS IN VALIDATION TEST. EXAMPLES IN FIG. 9.

\begin{tabular}{ccccccc}
\hline Case & Data ID & $\begin{array}{c}\text { Time } \\
\text { (YYYY-MM-DD hh:mm) }\end{array}$ & $\begin{array}{c}\text { Specular Point } \\
\text { (Lon, Lat) [deg] }\end{array}$ & $\begin{array}{c}\text { ASCAT Wind Speed } \\
{[\mathrm{m} / \mathrm{s}]}\end{array}$ & $\begin{array}{c}\text { Elevation Angle } \\
{[\mathrm{deg}]}\end{array}$ & $\begin{array}{c}\text { Antenna Gain } \\
{[\mathrm{dBic}]}\end{array}$ \\
\hline \multirow{2}{*}{32} & $\begin{array}{c}\text { RD000006/ } \\
\text { TD000148 }\end{array}$ & $2014-10-31$ T09:41 & $(-11.6,-47.1)$ & 10.5 & 81.2 & 12 \\
39 & $\begin{array}{c}\text { RD000007/ } \\
\text { TD000086 }\end{array}$ & $2014-11-08 \mathrm{~T} 15: 38$ & $(-105.5,-57.5)$ & 12.7 & 87.2 & 13 \\
\hline
\end{tabular}
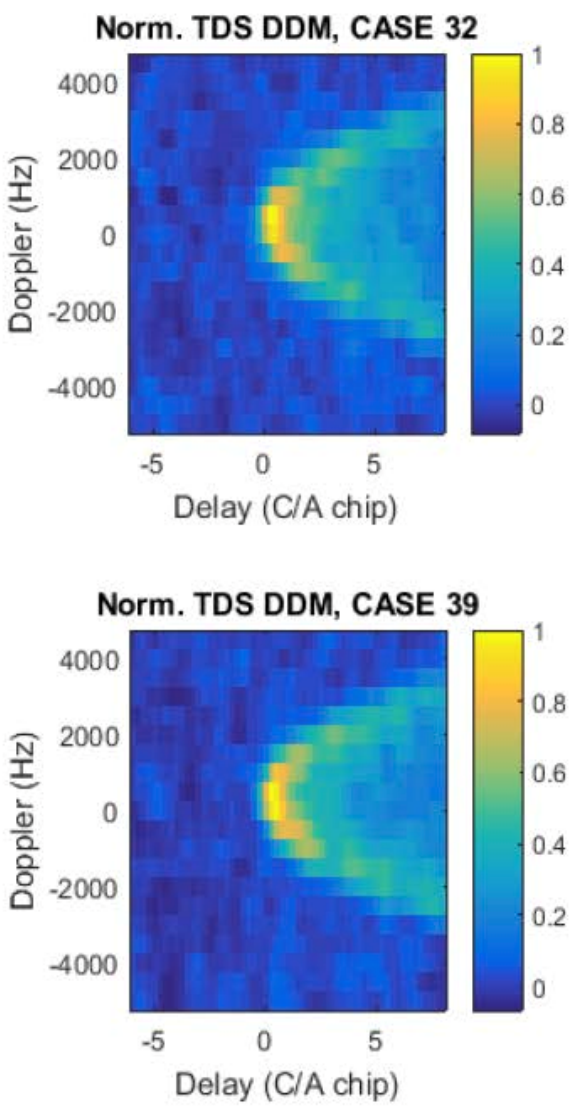
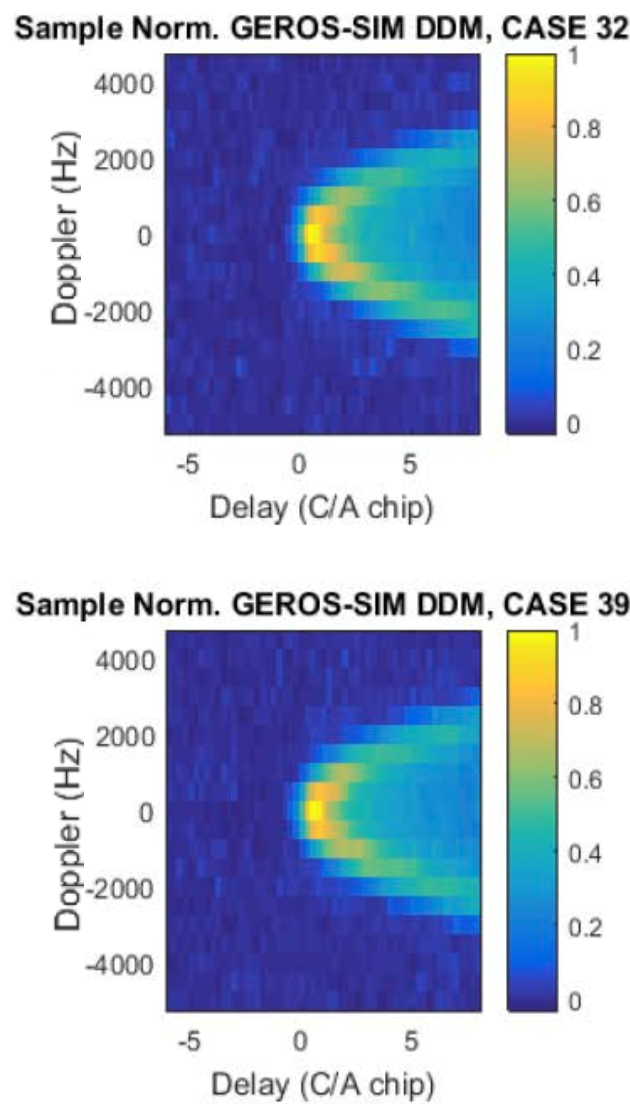

CASE:32/ ASCAT Wind $(\mathrm{m} / \mathrm{s}): 10.5$

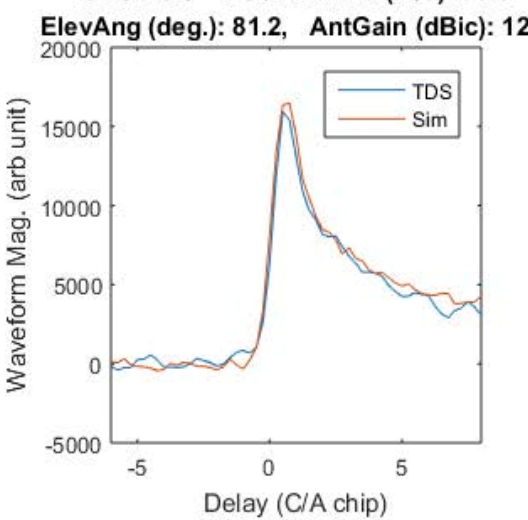

CASE:39/ ASCAT Wind $(\mathrm{m} / \mathrm{s}): 12.7$ ElevAng (deg.): 87.2, AntGain (dBic): 13

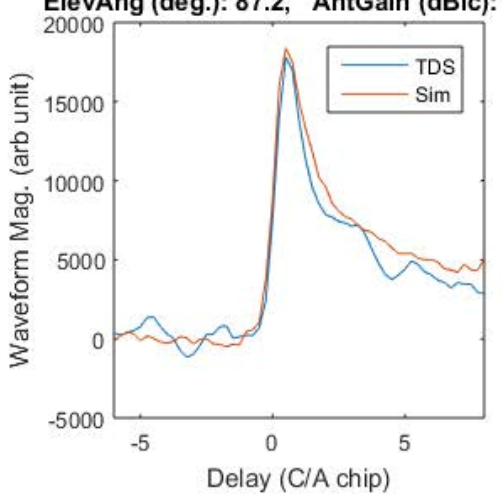

Fig. 9. Examples of GEROS-SIM validation test results comparing to TDS-1 data: (upper) Test Case 32 of $10.5 \mathrm{~m} / \mathrm{s}$ wind speed, (lower) Test Case 39 of $12.7 \mathrm{~m} / \mathrm{s}$ wind speed. The first column shows normalized DDM of TDS-1 data. The second column shows the normalized DDM simulated. The third column shows the comparison of the TDS- 1 and the simulated waveforms.

DDM/Waveforms and those of TDS-1. For the normalized waveform comparisons, the Mean Squared Error (MSE) in percentage is $12.76 \%$ in the range of delay $(-1-8)$ C/A chips. For the DDM peak comparison test, MSE in percentage is 16 $\%$ [58].

The simulated DDMs follows well the appearance of the actual measurements. The peak power of DDM shows also the characteristics in terms of wind speed, antenna gain, and elevation angle.The simulated DDM peaks decrease with high wind speed, similarly to the TDS-1 data. In Fig. 10, the peak power of TDS-1 and simulated DDM are compared. For the peak power with similar antenna gain in Fig. 10(b), this relation reveals clearly.

For the antenna gain, the cases of higher gain show the higher DDM peak both in TDS-1 and simulation results, as shown in Fig. 11(a). For the elevation angle, the cases of higher elevation show the higher peak, as shown in Fig. 11(b). Despite the higher elevation angle of CASE 24, the peak power is lower than the cases of lower elevation angles in 11(b). These show that the DDM simulation follows well the real measured DDM showing physically meaningful results.

In [58], the GEROS-SIM L1 generator is validated further more with L2 data generation (sea surface height and wind speed retrieval) in the GARCA project. The retrieval algorithms are tested and validated by using simulated L1 DDM and real measured data, e.g., TDS- 1 and airborne data. It means that the simulated L1 DDMs are satisfied in the validation test quantitatively and qualitatively, which are acceptable for development and validate the retrieval algorithm. The detailed validation test results of total GARCA/GEROS-SIM including retrieval algorithm and assessment are illustrated in [58]. 

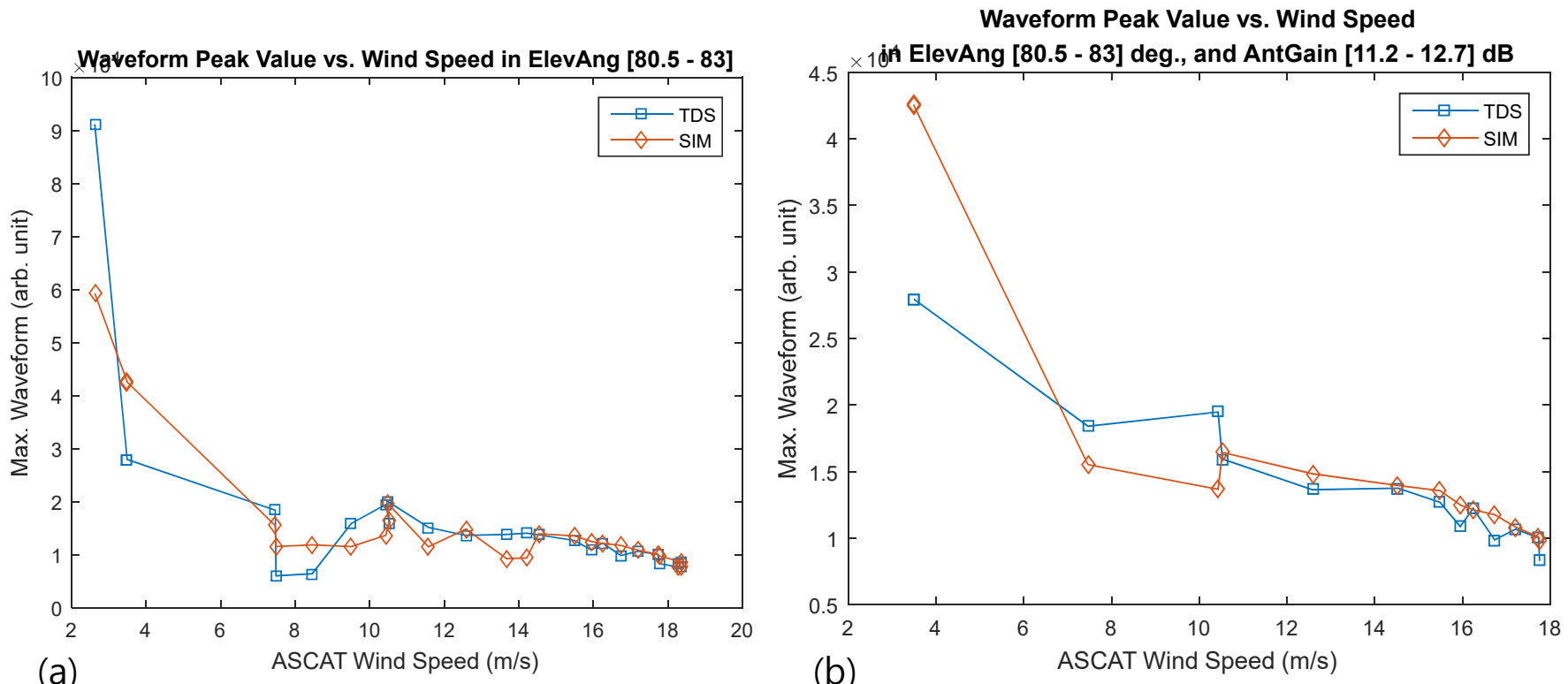

Fig. 10. DDM peak power comparison of GEROS-SIM validation test in terms of wind speed: (a) filtered by the elevation angle, and (b) filtered more by antenna gain. Peak values are displayed in linear scale.

\section{CONCLUSIONS}

This paper has presented the development of a complete L1 data simulator for generic GNSS-R spaceborne missions, such as GEROS-ISS. The framework and implementation of simulation is described in general way, and therefore it is helpful for development of other GNSS-R instrument simulator as well. As an instrument simulator, GEROS-SIM L1 can simulate the GNSS-R measurements including the instrument effects, scattering model, etc. It has been verified and validated with actual spaceborne and airborne GNSS$\mathrm{R}$ data in the GARCA Project. The simulated DDM shows the influence of simulation parameters such as wind speed, antenna gain, and elevation angle. The L1 simulator has been further validated via the retrieval algorithm test which used the simulated L1 and the real data. It enables accurate representation of measurements with respect to the observation geometry, geophysical parameters, and instrument parameters.

The GEROS-SIM L1 has a user-friendly interface for parameter input, and users can conveniently simulate and analyze the DDMs and waveforms in various observation scenarios. The current version of GEROS-SIM L1 is available on the web (http://www.tsc.upc.edu/rslab/gerossim) for public use [59]. This GNSS-R L1 simulator can be used in a wide range of GNSS-R research and development including instrument performance, mission analysis, altimeter and scatterometer studies, and geophysical parameter retrieval methods. Beyond the ocean reflectometry, the upgrade of GEROS L1 simulator is ongoing for Land reflection [60].

\section{REFERENCES}

[1] M. Martin-Neira, "A Passive Reflectometry and Interferometry System(PARIS)- Application to ocean altimetry," ESA J., vol. 17, no. 4, pp. 331-355, 1993.
[2] M. Martin-Neira, M. Caparrini, J. Font-Rossello, S. Lannelongue, and C. Vallmitjana, "The PARIS concept: an experimental demonstration of sea surface altimetry using GPS reflected signals," Geosci. Remote Sensing, IEEE Trans., vol. 39, no. 1, pp. 142-150, 2001.

[3] S. D'Addio and M. Martin-Neira, "Advanced paris altimeter based on delay compensation of Doppler waveforms," in Int. Geosci. Remote Sens. Symp., vol. 2, 2009.

[4] A. Rius, E. Cardellach, and M. Martin-Neira, "Altimetric Analysis of the Sea-Surface GPS-Reflected Signals," Geosci. Remote Sensing, IEEE Trans., vol. 48, no. 4, pp. 2119-2127, 2010.

[5] V. U. Zavorotny and A. G. Voronovich, "Scattering of GPS signals from the ocean with wind remote sensing application," IEEE Trans. Geosci. Remote Sens., vol. 38, no. 2, pp. 951-964, mar 2000.

[6] A. Rius, J. M. Aparicio, E. Cardellach, M. Martin-Neira, and B. Chapron, "Sea surface state measured using GPS reflected signals," Geophys. Res. Lett., vol. 29, no. 23, pp. art. no.-2122, 2002.

[7] J. Garrison, A. Komjathy, V. Zavorotny, and S. Katzberg, "Wind speed measurement using forward scattered GPS signals," IEEE Trans. Geosci. Remote Sens., vol. 40, no. 1, pp. 50-65, 2002.

[8] F. Soulat, M. Caparrini, O. Germain, P. Lopez-Dekker, M. Taani, and G. Ruffini, "Sea state monitoring using coastal GNSS-R," Geophys. Res. Lett., vol. 31, no. 21, 2004.

[9] S. Gleason, S. Hodgart, Y. Sun, C. Gommenginger, S. Mackin, M. Adjrad, and M. Unwin, "Detection and Processing of bistatically reflected GPS signals from low Earth orbit for the purpose of ocean remote sensing," IEEE Trans. Geosci. Remote Sens., vol. 43, no. 6, pp. 1229-1241, 2005.

[10] E. Cardellach and A. Rius, "A new technique to sense non-Gaussian features of the sea surface from L-band bi-static GNSS reflections," Remote Sens. Environ., vol. 112, no. 6, pp. 2927-2937, 2008.

[11] M. P. Clarizia, C. P. Gommenginger, S. T. Gleason, M. A. Srokosz, C. Galdi, and M. D. Bisceglie, "Analysis of GNSS-R delay-Doppler maps from the UK-DMC satellite over the ocean," jan 2009.

[12] A. Camps, X. Bosch-Lluis, I. Ramos-Perez, J. F. Marchán-Hernández, N. Rodríguez, E. Valencia, J. M. Tarongi, A. Aguasca, and R. Acevo, "New Passive Instruments Developed for Ocean Monitoring at the Remote Sensing LabUniversitat Politècnica de Catalunya," Sensors, vol. 9, no. 12, pp. 10 171-10 189, dec 2009.

[13] J. Marchan-Hernandez, E. Valencia, N. Rodriguez-Alvarez, I. RamosPerez, X. Bosch-Lluis, A. Camps, F. Eugenio, and J. Marcello, "Sea-State Determination Using GNSS-R Data," Geosci. Remote Sens. Lett. IEEE, vol. 7, no. 4, pp. 621-625, 2010.

[14] E. Valencia, A. Camps, J. Marchan-Hernandez, N. Rodriguez-Alvarez, I. Ramos-Perez, and X. Bosch-Lluis, "Experimental Determination of the 


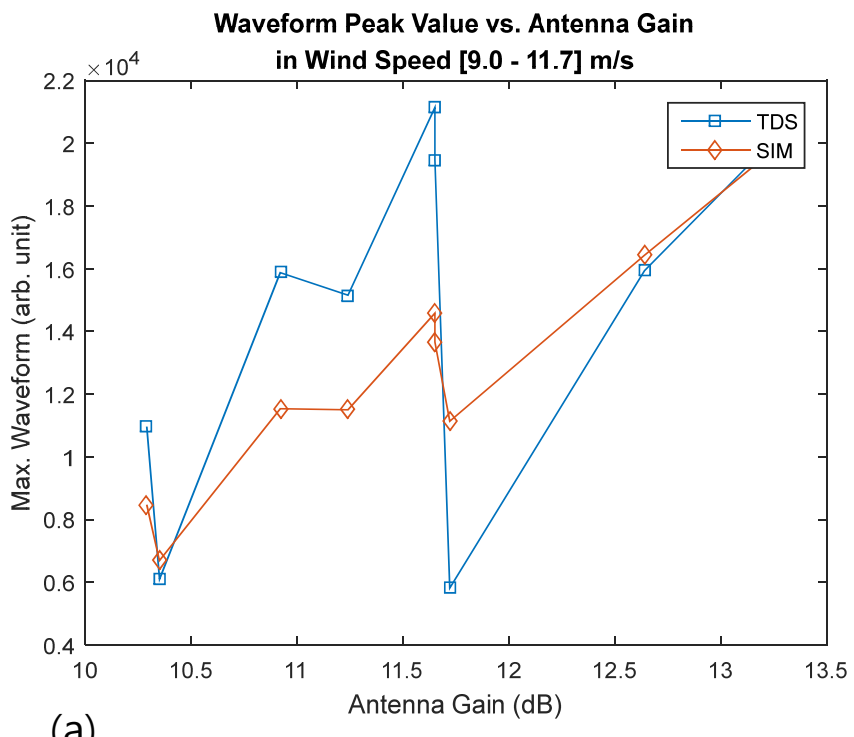

(a)

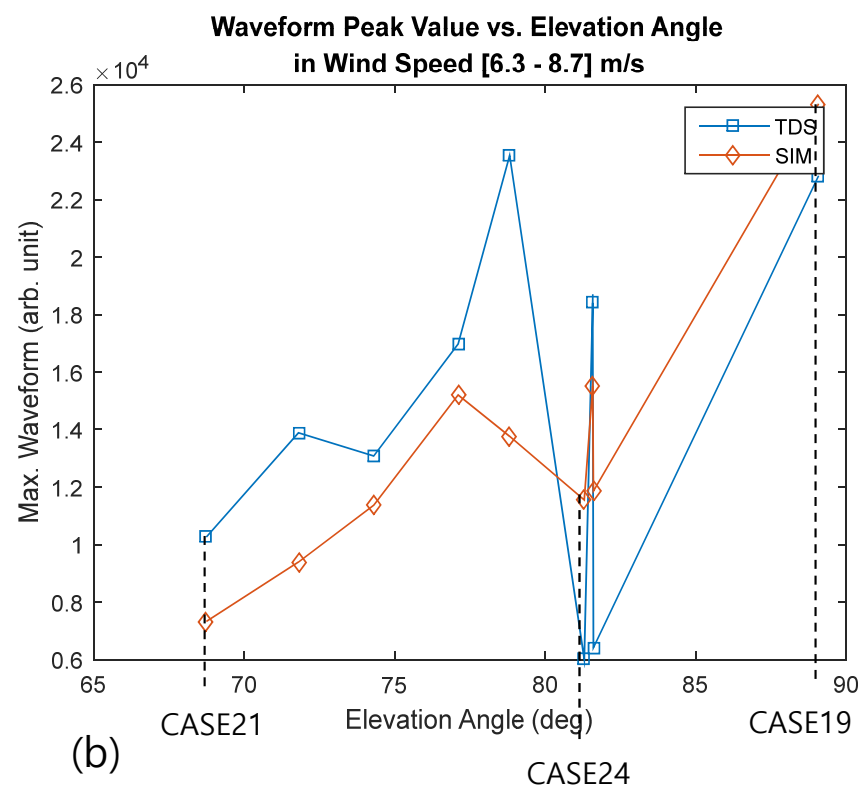

CASE24

Fig. 11. DDM peak power comparison of GEROS-SIM validation test in terms of (a) antenna gain filtered by the wind speed, and (b) elevation angle filtered by the wind speed. In (b) the antenna gains of CASE 21, 24 and 29 are 10.1, 10,2 and 12.7 dBic, respectively. Peak values are displayed in linear scale.

Sea Correlation Time Using GNSS-R Coherent Data," Geosci. Remote Sens. Lett. IEEE, vol. 7, no. 4, pp. 675-679, 2010.

[15] A. Komjathy, J. Maslanik, V. Zavorotny, P. Axelrad, and S. Katzberg, "Sea ice remote sensing using surface reflected GPS signals," vol. 7, 2000, pp. 2855-2857 vol.7.

[16] S. Gleason, "Towards Sea Ice Remote Sensing with Space Detected GPS Signals: Demonstration of Technical Feasibility and Initial Consistency Check Using Low Resolution Sea Ice Information,” Remote Sens., vol. 2, no. 8, pp. 2017-2039, aug 2010.

[17] A. AlonsoArroyo, V. U. Zavorotny, and A. Camps, "Sea ice detection using GNSS-R data from UK TDS-1," in 2016 IEEE Int. Geosci. Remote Sens. Symp. IEEE, jul 2016, pp. 2001-2004.

[18] S. J. Katzberg, O. Torres, M. S. Grant, and D. Masters, "Utilizing calibrated GPS reflected signals to estimate soil reflectivity and dielectric constant: Results from SMEX02," Remote Sens. Environ., vol. 100, no. 1, pp. 17-28, jan 2006.

[19] A. Alonso-Arroyo, A. Camps, A. Monerris, C. Rudiger, J. P. Walker, R. Onrubia, J. Querol, H. Park, and D. Pascual, "On the Correlation Between GNSS-R Reflectivity and L-Band Microwave Radiometry," IEEE J. Sel. Top. Appl. Earth Obs. Remote Sens., pp. 1-18, 2016.

[20] M. Unwin, P. Jales, J. Tye, C Gommenginger, G. Foti, and J. Rosello, "Spaceborne GNSS-Reflectometry on TechDemoSat-1: Early Mission Operations and Exploitation," IEEE J. Sel. Top. Appl. Earth Obs. Remote Sens., vol. 9, no. 10, pp. 4525-4539, 2016.

[21] J. Wickert, E. Cardellach, M. Martin-Neira, J. Bandeiras, L. Bertino, O. B. Andersen, A. Camps, N. Catarino, B. Chapron, F. Fabra, N. Floury, G. Foti, C. Gommenginger, J. Hatton, P. Hoeg, A. Jaggi, M. Kern, T. Lee, Z. Li, H. Park, N. Pierdicca, G. Ressler, A. Rius, J. Rosello, J. Saynisch, F. Soulat, C. K. Shum, M. Semmling, A. Sousa, J. Xie, and C. Zuffada, "GEROS-ISS: GNSS REflectometry, Radio Occultation, and Scatterometry Onboard the International Space Station," IEEE J. Sel. Top. Appl. Earth Obs. Remote Sens., vol. 9, no. 10, pp. 4552-4581, oct 2016.

[22] C. Ruf, M. Unwin, J. Dickinson, R. Rose, D. Rose, M. Vincent, and A. Lyons, "CYGNSS: Enabling the Future of Hurricane Prediction [Remote Sensing Satellites]," IEEE Geosci. Remote Sens. Mag., vol. 1, no. 2, pp. 52-67, jun 2013.

[23] R. Olive, A. Amezaga, H. Carreno-Luengo, H. Park, and A. Camps, "Implementation of a GNSS-R Payload Based on Software-Defined Radio for the 3CAT-2 Mission," IEEE J. Sel. Top. Appl. Earth Obs. Remote Sens., vol. 9, no. 10, pp. 4824-4833, oct 2016

[24] H. Carreno-Luengo, A. Camps, P. Via, J. F. Munoz, A. Cortiella, D. Vidal, J. Jane, N. Catarino, M. Hagenfeldt, P. Palomo, and S. Cornara, "3Cat-2An Experimental Nanosatellite for GNSS-R Earth Observation: Mission Concept and Analysis," IEEE J. Sel. Top. Appl. Earth Obs. Remote Sens., vol. 9, no. 10, pp. 4540-4551, oct 2016.
[25] J. Garrison, " A statistical model and simulator for ocean-reflected GNSS signals," IEEE Trans. Geosci. Remote Sens., vol. 54, no. 10 pp. 60076019, 2016.

[26] G. Giangregorio, M. di Bisceglie, P. Addabbo, T. Beltramonte, S. DAddio, and C. Galdi, "Stochastic Modeling and Simulation of DelayDoppler Maps in GNSS-R Over the Ocean," IEEE Trans. Geosci. Remote Sens., vol. 54, no.4 pp. 2056-2069, 2016.

[27] M. Martin-Neira, S. D'Addio, C. Buck, N. Floury, and R. PrietoCerdeira, "The PARIS Ocean Altimeter In-Orbit Demonstrator," IEEE Trans. Geosci. Remote Sens., vol. 49, no. 6, pp. 2209-2237, jun 2011.

[28] H. Park, J. F. J. Marchan-Hernandez, N. Rodriguez-Alvarez, E. Valencia, I. Ramos-Perez, X. Bosch-Lluis, and A. Camps, "End-to-end simulator for Global Navigation Satellite System Reflectometry space mission," in 2010 IEEE Int. Geosci. Remote Sens. Symp. IEEE, jul 2010, pp. 42944297.

[29] A. Camps, I. Corbella, M. Vall-llossera, N. Duffo, F. Marcos, F. Martinez-Fadrique, and M. Greiner, "The SMOS end-to-end performance simulator: description and scientific applications," vol. 1, 2003, pp. $13-15$ vol.1.

[30] J. Marchan-Hernandez, A. Camps, N. Rodriguez-Alvarez, E. Valencia, X. Bosch-Lluis, and I. Ramos-Perez, "An Efficient Algorithm to the Simulation of Delay-Doppler Maps of Reflected Global Navigation Satellite System Signals," IEEE Trans. Geosci. Remote Sens., vol. 47, no. 8, pp. 2733-2740, 2009.

[31] H. You, J. Garrison, G. Heckler, and V. Zavorotny, "Stochastic voltage model and experimental measurement of ocean-scattered GPS signal statistics," IEEE Trans. Geosci. Remote Sens., vol. 42, no. 10, pp. 2160 2169,2004

[32] A. Camps, X. Bosch-Lluis, and H. Park, "IMPACT OF RECEIVER'S FREQUENCY RESPONSE IN GNSS REFLECTOMETERS," in 2010 IEEE Int. Geosci. Remote Sens. Symp. IEEE, jul 2010, pp. 3817-3820.

[33] H. Park, A. Camps, E. Valencia, N. Rodriguez-Alvarez, X. Bosch-Lluis, I. Ramos-Perez, and H. Carreno-Luengo, "Retracking considerations in spaceborne GNSS-R altimetry," GPS Solut., vol. 16, no. 4, pp. 507-518, 2012.

[34] H. Park, E. Valencia, A. Camps, A. Rius, S. Ribo, and M. Martin-Neira, "Delay Tracking in Spaceborne GNSS-R Ocean Altimetry," Geosci. Remote Sens. Lett. IEEE, vol. 10, no. 1, pp. 57-61, jan 2013.

[35] C. Cox and W. Munk, "Measurement of the Roughness of the Sea Surface from Photographs of the Suns Glitter," J. Opt. Soc. Am., vol. 44, no. 11, pp. 838-850, nov 1954.

[36] E. Cardellach, and A. Rius, "A new technique to sense non-Gaussian features of the sea surface from L-band bi-static GNSS reflections," Remote Sens. Environ., vol. 112, no. 6, pp. 2927-2937, 2008. 
[37] S. J. Katzberg, O. Torres, and G. Ganoe, "Calibration of reflected GPS for tropical storm wind speed retrievals," Geophys. Res. Lett., vol. 33, p. 5 PP., sep 2006.

[38] J. A. Klobuchar, "Ionospheric effects on GPS," GPS World, vol. 2, no. 4, pp. 48- 51, 1991.

[39] A. Camps, H. Park, G. Foti, and C. Gommenginger, "Ionospheric Effects in GNSS-Reflectometry From Space," IEEE J. Sel. Top. Appl. Earth Obs. Remote Sens., pp. 1-11, 2016.

[40] B. Nava, S. Radicella, R. Leitinger, and P. Coïsson, "Use of total electron content data to analyze ionosphere electron density gradients," Adv. Sp. Res., vol. 39, no. 8, pp. 1292-1297, 2007.

[41] "Trans-ionospheric radio propagation: The global ionospheric scintillation model (GISM)." [Online]. Available: http://www.itu.int/pub/R-REPP. 2097-2007

[42] J. J. Spilker, "Tropospheric effects on GPS," in Glob. Position. Syst. Theory Appl., 1996, pp. 517-546.

[43] Roual Observatory of Belgium, GNSS Research Group, "Tropospheric delay and GNSS signals: Tutorial,” Tech. Rep., 2012. [Online]. Available: http://gnss.be

[44] F. K. Brunner and W. M. Welsch, "Effect of the troposphere on GPS measurements," GPS World, vol. 4, no. 1, pp. 42-46, 1993.

[45] X. Bosch-Lluis, I. Ramos-Perez, A. Camps, N. Rodriguez-Alvarez, J. Marchan-Hernandez, E. Valencia, J. Nieto, and M. Guerrero, "Digital beamforming analysis and performance for a digital L-band Pseudocorrelation radiometer," in 2009 IEEE Int. Geosci. Remote Sens. Symp. IEEE, 2009, pp. V-184-V-187.

[46] A. J. Carmona, "Application of Interferometric Radiometry to Earth Observation," Ph.D. dissertation, 1997.

[47] D. Pascual (2017, Mar.) GNSS-matlab, [Online]. Available:http://http //rscl-grss.org/coderecord.php?id=479, doi: 10.21982/M8F59K

[48] D. Pascual, S. Member, H. Park, A. Camps, A. A. Arroyo, and R. Onrubia, "Simulation and Analysis of GNSS-R Composite Waveforms Using GPS and Galileo Signals," IEEE J. Sel. Top. Appl. Earth Obs. Remote Sens., vol. 7, no. 5, pp. 1461-1468, 2014.

[49] R. Raney and G. Wessels, "Spatial considerations in SAR speckle consideration," IEEE Trans. Geosci. Remote Sens., vol. 26, no. 5, pp. $666-672,1988$.

[50] R. Touzi, "A review of speckle filtering in the context of estimation theory," IEEE Trans. Geosci. Remote Sens., vol. 40, no. 11, pp. 23922404, 2002

[51] M. P. Clarizia, C. Gommenginger, M. Di Bisceglie, C. Galdi, and M. Srokosz, "Simulation of L-Band Bistatic Returns From the Ocean Surface: A Facet Approach With Application to Ocean GNSS Reflectometry," IEEE Trans. Geosci. Remote Sens., vol. PP, no. 99, pp. 1-12.

[52] H. Park, A. Camps, D. Pascual, A. Alonso, F. Martin, and H. CarrenoLuengo, " Improvement of the PAU/PARIS End-to-end Performance Simulator (P2EPS) in preparation for upcoming GNSS-R missions," in 2013 IEEE Int. Geosci. Remote Sens. Symp. - IGARSS. IEEE, jul 2013, pp. 362-365.

[53] H. Park, A. Camps, D. Pascual, A. Alonso-Arroyo, F. Martin, H. Carreno-Luengo, and R. Onrubia, "Simulation study on tropicial cyclone tracking from the ISS using GNSS-R measurements," in 2014 IEEE Geosci. Remote Sens. Symp. IEEE, jul 2014, pp. 4062-4065.

[54] H. Park, A. Camps, D. Pascual, R. Onrubia, A. Alonso-Arroyo, and F. Martin, " Evolution of PAU/PARIS End-to-end Performance Simulator $\left(\mathrm{P}^{2}\right.$ EPS) towards GNSS reflectometry, radio occulation and Scatterometry simulator (GEROS-SIM)," in 2015 IEEE Int. Geosci. Remote Sens. Symp. IEEE, jul 2015, pp. 4757-4760.

[55] H. Park, D. Pascual, F. Martín, E. Valencia, and A. Camps, "ID-17A : Contribution to system end-to-end performance model and validation report,” Tech. Univ. Catalonia, Barcelona, Spain, Tech. Rep. PARISPhAIEEC-UPC-TN-11, 2012.

[56] C. Gommenginger et al., "GARCA Technical Note 3: Assessment of Requirements and Consolidation of Retrieval Algorithms," ESA/ESTEC, Noordwijk, The Netherlands, Tech. Rep. ESA-AO1-7850/14-GARCATN-3, 2016. Available:ftp://ftp.gfz-potsdam.de/pub/home/kg/incoming/ GARCA/DOCUMENTS/TECHNICAL_NOTES/GARCA_TN3.pdf

[57] G. Foti, C. Gommenginger, P. Jales, M. Unwin, A. Shaw, C. Robertson, and, J. "Spaceborne GNSS reflectometry for ocean winds: First results from the UK TechemoSat-1 mission," Geophys. Res. Lett., vol. 42, no. 13, pp. 54355441, Jul. 2015.

[58] E. Cardellach et al., "GARCA Technical Note 4: Data Acquisition and Analysis Report," ESA/ESTEC, Noordwijk, The Netherlands, Tech. Rep. ESA-AO1-7850/14-GARCA-TN-4, 2016. Available:ftp://ftp.gfz-potsdam.de/pub/home $/ \mathrm{kg} /$ incoming/GARCA/ DOCUMENTS/TECHNICAL_NOTES/GARCA_TN4.pdf
[59] H. Park, A. Camps, D. Pascual, Y. Kang, and R. Onrubia, "GARCA/GEROS-SIM M2 (Instrument to L1 module) Web Online Simulation Tool," RSCL, [Onlnie] Available:http://rscl-grss.org/coderecord. php?id=474

[60] H. Park, A. Camps, D. Pascual, A. Alonso-Arroyo, J. Querol, and R. Onrubia, "Improvement of PAU/PARIS end-to-end performance simulator (P2EPS): Land scattering including topography," in 2016 IEEE Int. Geosci. Remote Sens. Symp. IEEE, jul 2016, pp. 5607-5610.

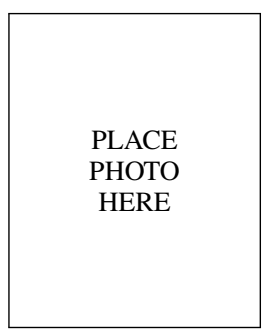

Michael Shell Biography text here.

John Doe Biography text here.

Jane Doe Biography text here. 